\title{
Carleman approximation on Riemannian manifolds by harmonic functions with Newtonian singularities
}

\author{
Pierre Blanchet \\ Centre de recherches mathématiques, Université de Montréal, Montréal, Canada
}

\section{Email address:}

vltcbqmj@yahoo.fr

\section{To cite this article:}

Pierre Blanchet. Carleman Approximation on Riemannian Manifolds by Harmonic Functions with Newtonian Singularities. American Journal of Applied Mathematics. Vol. 3, No. 1, 2015, pp. 1-3. doi: 10.11648/j.ajam.20150301.11

Abstract: In 1927, it was proved by Carleman that the real line was a set of Carleman approximation by entire functions. In this paper, the analogous problem for harmonic approximation on Riemannian manifolds is discussed.

Keywords: Harmonic Functions, Harmonic Approximation, Newtonian Functions, Carleman Approximation, Riemannian Manifolds

\section{Introduction}

In its classical form, the Theorem of Carleman states that any continuous function on the real line of the complex plane can be approximated on this straight line by an entire function with an error decreasing at an arbitrary speed. This article is devoted to the same problem for harmonic approximation by Newtonian functions on closed subsets of Riemannian manifolds.

\section{Riemannian Manifolds}

Let $\Omega$ denote a connected $\mathrm{C}^{\infty}$ manifold. If $\mathrm{V}$ is a coordinate region in $\Omega$ with coordinates $\mathrm{x}=\left(\mathrm{x}^{1}, \ldots, \mathrm{x}^{\mathrm{n}}\right)$, and $\tilde{V}$ is an overlapping coordinate region with coordinates $\tilde{\mathrm{x}}=\left(\tilde{\mathrm{x}}^{1}, \ldots, \tilde{\mathrm{x}}^{\mathrm{n}}\right)$, we then obtain a $\mathrm{C}^{\infty}$ map $\mathrm{x} \rightarrow \tilde{\mathrm{x}}$ whose Jacobian matrix $\partial \tilde{x} / \partial x$ has $i j{ }^{\text {th }}$ entry equal to $\partial \tilde{x}^{i} / \partial x^{j}$. We assume that $\Omega$ is orientable, which means that we may select a family of coordinate regions covering $\Omega$ such that for every pair of overlapping coordinate regions $\mathrm{V}$ and $\tilde{\mathrm{V}}$ from the family we have $\operatorname{det}(\partial \tilde{\mathrm{x}} / \partial \mathrm{x})>0$; for the rest of this paper we suppose a maximal family of coordinate regions with this property is fixed, and all coordinate regions are understood to be from this family. If $\mathrm{A}$ is any subset of $\Omega$, then $\mathrm{C}$ (A) denotes the set of all continuous real-valued functions on $\mathrm{A}$. If $\mathrm{V} \subset \Omega$ is open, then functions in $\mathrm{C}^{\infty}(\mathrm{V})$ are called smooth; we let $\mathrm{C}_{0}(\mathrm{~V})\left[\right.$ resp. $\left.\mathrm{C}_{0}^{\infty}(\mathrm{V})\right]$ denote the set of all functions $\mathrm{u} \in \mathrm{C}(\mathrm{V})\left[\right.$ resp. $\mathrm{u} \in \mathrm{C}^{\infty}(\mathrm{V})$ ] which vanish off a compact subset of $\mathrm{V}$. If a local coordinate mapping carries a subregion of $\Omega$ onto a subregion of $\mathrm{R}^{\mathrm{n}}$ containing a closed ball $\left\{\mathrm{x} \in \mathrm{R}^{\mathrm{n}}:\left|\mathrm{x}-\mathrm{x}_{0}\right| \leq \mathrm{r}\right\}, r>0$, then the preimage of the open ball $\left\{x \in R^{n}:\left|x-x_{0}\right|<r\right\}$ is called a parametric ball in $\Omega$.

We assume that $\Omega$ is a Riemannian manifold, which means that at each point $\mathrm{p} \in \Omega$ the tangent space $\mathrm{T}_{\mathrm{p}}(\Omega)$ carries an inner product $\mathrm{g}_{\mathrm{p}}(\cdot, \cdot)$, and for each pair of smooth vector fields $\mathrm{X}$ and $\mathrm{Y}$, the function $\Omega \ni \mathrm{p} \rightarrow \mathrm{g}_{\mathrm{p}}(\mathrm{X}(\mathrm{p}), \mathrm{Y}(\mathrm{p}))$ is smooth. We will use the notation $X \cdot Y \equiv g_{p}(X(p), Y(p))$. If $X$ is a smooth vector field, then $|X| \equiv \sqrt{X \cdot X}$ is a well-defined continuous function, and is smooth on the open set where it is strictly positive. In each local coordinate system we write

$$
g_{i j}=g_{i j}(p)=g_{p}\left(\frac{\partial}{\partial x^{i}}, \frac{\partial}{\partial x^{j}}\right) ;
$$

and we will let $\left(\mathrm{g}^{\mathrm{ij}}\right)$ and $\mathrm{g}$ denote the inverse matrix and the determinant of the positive definite symmetric matrix $\left(\mathrm{g}_{\mathrm{ij}}\right)$.

From this definition we see that $\left(g_{i j}\right)$ is a covariant tensor of order 2, which means that for overlapping coordinate regions $\mathrm{V}$ and $\tilde{\mathrm{V}}$ we have

$$
\left(\tilde{\mathrm{g}}_{\alpha \beta}\right)=\left(\frac{\partial \mathrm{x}}{\partial \tilde{\mathrm{x}}}\right)^{\mathrm{T}} \cdot \mathrm{g}_{\mathrm{ij}} \cdot \frac{\partial \mathrm{x}}{\partial \tilde{\mathrm{x}}} .
$$

By taking determinants in this formula we obtain 


$$
\sqrt{\tilde{\mathrm{g}}}=\sqrt{\mathrm{g}} \operatorname{det} \frac{\partial \mathrm{x}}{\partial \tilde{\mathrm{x}}}
$$

If $\mathrm{u} \in \mathrm{C}_{0}(\mathrm{~V} \cap \tilde{\mathrm{V}})$, then from this and the change-of-variables formula we obtain

$$
\int \mathrm{u} \sqrt{\mathrm{g}} \mathrm{dx}{ }^{1} \cdots \mathrm{dx} \mathrm{x}^{\mathrm{n}}=\int \mathrm{u} \sqrt{\widetilde{g}} \mathrm{~d} \tilde{\mathrm{x}}^{1} \cdots \mathrm{d} \tilde{\mathrm{x}}^{\mathrm{n}}
$$

and this common integral is denoted $\int \mathrm{ud} \lambda$. It follows that by use of finite partitions of unity we may give a natural definition of $\int \mathrm{ud} \lambda$ for every $\mathrm{u} \in \mathrm{C}_{0}(\Omega)$. This defines a positive linear functional on $\mathrm{C}_{0}(\Omega)$, which corresponds to a positive measure $\lambda$ on $\Omega$ called the volume measure; we denote the set of $\lambda$-integrable functions on an open set $\mathrm{W} \subset \Omega$ by $\mathrm{L}_{1}(\mathrm{~W})$, and the locally $\lambda$-integrable functions on $\mathrm{W}$ by $\mathrm{L}_{1}(\mathrm{~W}, \mathrm{loc})$.

A relatively compact region $R \subset \Omega$ is said to be a regular subregion of $\Omega$ provided that $\partial R$ is nonempty and for each point $p \in \partial R$ there exists a parametric region $V \ni p$, with local coordinates $\left(\mathrm{x}^{1}, \ldots, \mathrm{x}^{\mathrm{n}}\right)$, such that the points of $\mathrm{V} \cap \partial \mathrm{R}$ correspond to $x^{n}=0$, and the points of $\mathrm{V} \cap \mathrm{R}$ correspond to $x^{n}<0$. Using parametric regions of this special type, with coordinates $\left(\mathrm{x}^{1}, \ldots, \mathrm{x}^{\mathrm{n}-1}\right)$ as local coordinates for $\partial R$, we see that $\partial R$ is a finite disjoint union of orientable manifolds. Note that for each point $p \in \partial R$, we may restrict the inner product $g_{p}$ to the tangent space of $\partial R$ at $p$; then each component of $\partial R$ becomes a Riemannian manifold, and the sum of the volume measures on these components is denoted $\sigma$.

If $\gamma:[\alpha, \beta] \rightarrow \Omega$ is a piecewise-smooth arc contained in a parametric ball, then the length

$$
\int_{\alpha}^{\beta}\left(g_{i j}(x(t)) \frac{d x^{i}}{d t} \frac{d x^{j}}{d t}\right)^{1 / 2} d t
$$

is invariantly defined, and by using finite partitions of unity one may define the length of any piecewise-smooth arc in $\Omega$. The distance between any two points of $\Omega$ is the infimum of the lengths of piecewise-smooth arcs joining the points. This gives a metric on $\Omega$ whose topology coincides with the manifold topology; from this it can be shown that $\Omega$ is second countable, and that there exist partitions of unity subordinate to any open cover of $\Omega$. If $\Omega$ is non-compact, it can be written as the union of a sequence of regular subregions $R_{1} \subset \subset R_{2} \subset \subset \cdots$.

If a smooth contravariant vector field on $\Omega$ is given locally by functions $a^{i}$, then there is a well-defined smooth function $\operatorname{div}$ a $: \Omega \rightarrow \mathrm{R}$ whose value in each system of local coordinates $\left(\mathrm{x}^{1}, \ldots, \mathrm{x}^{\mathrm{n}}\right)$ is given by the expression

$$
\operatorname{div} a=\frac{1}{\sqrt{g}} \sum_{i=1}^{n} \frac{\partial\left(\sqrt{g} a^{i}\right)}{\partial x^{i}}
$$

Let $\mathrm{V} \subset \Omega$ be open. The Laplace operator $\Delta: \mathrm{C}^{\infty}(\mathrm{V}) \rightarrow \mathrm{C}^{\infty}(\mathrm{V})$ is defined by $\Delta u=\operatorname{div}(\operatorname{grad} u)$. In local coordinates,

$$
\Delta u=\frac{1}{\sqrt{g}} \sum_{i, j=1}^{n} \frac{\partial}{\partial x^{i}}\left(\sqrt{g} g^{i j} \frac{\partial u}{\partial x^{j}}\right) .
$$

We say that $\mathrm{u} \in \mathrm{C}^{\infty}(\mathrm{V})$ is harmonic in $\mathrm{V}$ provided that $\Delta \mathrm{u} \equiv 0$ in $\mathrm{V}$.

If $R$ is a regular subregionof $\Omega$, then the Green function of $R$ is a symmetric function $\mathrm{G}_{\mathrm{R}}: \mathrm{R} \times \mathrm{R} \rightarrow(0, \infty]$ with the following properties :

(a) If $q \in R$, then the function $G_{R}(\cdot, q)$ is harmonic on $\mathrm{R} \backslash\{q\}$, continuously assumes the value $+\infty$ at $q$, and approaches zero at $\partial R$.

(b) $G_{R}$ is continuous on $\{(\mathrm{p}, \mathrm{q}) \in \mathrm{R} \times \mathrm{R}: \mathrm{p} \neq \mathrm{q}\}$.

(c) If $q \in \mathrm{R}$, then $\mathrm{G}_{\mathrm{R}}(\cdot, \mathrm{q}) \in \mathrm{L}_{1}(\mathrm{R})$ and $\varphi(\mathrm{q})=-\int_{\mathrm{R}} \mathrm{G}_{\mathrm{R}}(\mathrm{p}, \mathrm{q}) \Delta \varphi(\mathrm{p}) \mathrm{d} \lambda(\mathrm{p})$ for all $\varphi \in \mathrm{C}_{0}^{\infty}(\mathrm{R})$.

Let $\Omega$ be an orientable Riemannian manifold. We say that a continuous function $\mathrm{u}: \Omega \rightarrow[-\infty, \infty]$ is Newtonian provided that there is a discrete set $\mathrm{A} \subset \Omega$ with the following properties:

i) $\left.\mathrm{u}\right|_{\Omega \backslash \mathrm{A}}$ is harmonic, and

ii)for each point $a \in A$ there is a regular subregion $R$ satisfying $\mathrm{R} \cap \mathrm{A}=\{\mathrm{a}\}$, and a constant $\mathrm{c}$, such that the function $\mathrm{u}-\mathrm{cG}_{\mathrm{R}}(., \mathrm{a})$ can be defined at aso as to be harmonic on $R$ where $G_{R}$ is the Green function of $R$.

It follows that if $\mathrm{u}: \Omega \rightarrow[-\infty, \infty]$ is Newtonian, and ais any point in any regular subregion $\mathrm{R}$, then there is a constant $\mathrm{c}$ such that the function $\mathrm{u}-\mathrm{cG}_{\mathrm{R}}(., \mathrm{a})$ is harmonic in a neighborhood of $\mathbf{a}$.

The following theorem was proved several years ago in [1, Theorem 5.1].

Theorem 1.(Runge theorem for Newtonian approximation)

Let $\Omega$ be an orientable Riemannian manifold. Let $F$ be a closed subset of $\Omega$, and let $\mathrm{f}$ be harmonic on an open set containing F. If $\in>0$, then there exists a Newtonian function h on $\Omega$ such that $\sup _{\mathrm{F}}|\mathrm{f}-\mathrm{h}|<\epsilon$.

If $F$ is a closed subset of $\Omega$, we say that a function $\mathrm{f} \in \mathrm{C}(\mathrm{F})$ is in the class $\mathrm{H}(\mathrm{F})$ provided that $\mathrm{f}$ can be extended to a harmonic function on some open set containing $\mathrm{F}$. We denote by $\overline{\mathrm{H}}(\mathrm{F})$ the set of all functions $\mathrm{f} \in \mathrm{C}(\mathrm{F})$ which can be uniformly approximated on $\mathrm{F}$ by functions in $\mathrm{H}(\mathrm{F})$ and we set $\mathrm{A}(\mathrm{F})=\mathrm{C}(\mathrm{F}) \cap \mathrm{H}($ int $\mathrm{F})$.

We consider a set $\mathrm{A} \subset \Omega$ and a point $\varphi \in \Omega$. We say that $\mathrm{A}$ is thin at $\varphi$ provided that either $\varphi \notin(\mathrm{A} \backslash\{\varphi\})^{-}$, or $\varphi \in(\mathrm{A} \backslash\{\varphi\})^{-}$and there exists a positive superharmonic 
function on a parametric ball $\mathrm{B} \ni \varphi$ such that

$$
\liminf _{\mathrm{A} \cap \mathrm{B} \ni \mathrm{x} \rightarrow \varphi} \mathrm{v}(\mathrm{x})>\mathrm{v}(\varphi) .
$$

The following theorem was proved in [1, Theorem 8.1].

Theorem 2.(Keldysh theorem for closed sets). Let F be a closed subset of an orientable Riemannian manifold $\Omega$. Then the following are equivalent:

(a) $\mathrm{A}(\mathrm{F})=\overline{\mathrm{H}}(\mathrm{F})$.

(b) $\Omega \backslash F$ and $\Omega \backslash$ int $F$ are thin at the same points.

Let $\Omega$ be an orientable Riemannian manifold and let $F$ be a closed subset of $\Omega$. Let $J$ be a class of functions defined on $\Omega$. We say that $\mathrm{F}$ is a set of Carleman approximation by functions in the class $\mathrm{J}$ if for each pair of functions $\mathrm{u}$ and $\in$ continuous on $\mathrm{F}$, with $\in$ positive, there exists a function $\mathrm{v}$ $\in \mathrm{J}$ satisfying

$$
|\mathrm{u}(\mathrm{x})-\mathrm{v}(\mathrm{x})|<\in(\mathrm{x}), \mathrm{x} \in \mathrm{F} .
$$

In 1927, it was proved by Carleman that the real axis was a set of Carleman approximation by entire functions.

In this paper, we consider the analogous problem for harmonic approximation on Riemannian manifolds.

Our purpose is to prove the following theorem.

Theorem 3. Let $\Omega$ be an orientable Riemannian manifold, and let $\mathrm{F}$ be a closed subset of $\Omega$ such that int $\mathrm{F}=\phi$. Then F is a set of Carleman approximation by Newtonian functions on $\Omega$ if and only if $\Omega \backslash F$ is thin at no point of $F$.

This theorem was proved by P.M. Gauthier in the case in which $\Omega$ is a domain in $\mathrm{R}^{\mathrm{n}}$, see [2].

\section{Proof of Theorem 3}

To prove the theorem we consider first the case where $\Omega$ is compact. The sufficiency in this case follows from the Keldysh Theorem, the Runge Theorem and the fact that the continuous function $\in$ reaches its minimum at a point of $\mathrm{F}$.

To prove the sufficiency in the case where $\Omega$ is non-compact, we take an exhaustion of $\Omega$ by regular domains and we apply iteratively (recursively) both Theorem 1 and Theorem 2 by means of a lemma of M. Labrèche, itself a consequence of the Keldysh Theorem and we conclude with a standard argument. The thinness condition of Theorem 3 allows applying that lemma to the problem at each step.

This method is inspired from the one of Carleman [3] in his proof and follows closely the same arguments with small modifications. In particular, the Keldysh Theorem is used to approximate the given function on suitable compact subsets at each iteration. This completes the proof of the sufficiency.

The necessity of Theorem 3 in both cases, compact or non-compact, follows at once from the fact that uniform approximation is weaker than Carleman approximation and Theorem 2, the Keldysh Theorem for closed sets. This completes the proof of the theorem. The reader is referred to the work of P.M. Gauthier for a more detailed account.

\section{Applicability}

As a result of the preceding development, our Theorem 3 which is the main novelty of this work, may be applied to any smooth curve and therefore to any geodesic on a Riemannian manifold. More generally, Carleman approximation theorems have wide applicability in pure and applied mathematics to several problems and are powerful tools to prove various new properties of harmonic functions. For more insight into this field the reader might consult the article of $\mathrm{T}$. Bagby and P.M.Gauthier [4] as well as the reports of the same authors in [5] and [6].

\section{References}

[1] T. Bagby and P. Blanchet, Uniform harmonic approximation on Riemannian manifolds, Journal d'AnalyseMathématique62 (1994), 47-76.

[2] P.M. Gauthier, Carleman approximation on unbounded sets by harmonic functions with Newtonian singularities, in Proceedings of the $8^{\text {th }}$ Conference on analytic functions, Blazejewko, Poland, August, 1982.

[3] T. Carleman, Sur un théorème de Weierstrass, Arkiv för Matematik, Astronomi och Fysik, Band 20B. N:0 4 (1927), 1-5.

[4] T. Bagby and P.M. Gauthier, Approximation by harmonic functions on closed subsets of Riemann surfaces, Journal d'AnalyseMathématique51 (1988), 259-284.

[5] P.M. Gauthier, Uniform approximation, in Complex Potential Theory, Université de Montréal (P.M. Gauthier Editor), Kluwer Academic Publishers, Dordrecht, The Netherlands, 1994, 235-271.

[6] T. Bagby and P.M. Gauthier, Harmonic approximation on closed subsets of Riemannian manifolds, in Complex Potential Theory,Université de Montréal (P.M. Gauthier Editor), Kluwer Academic Publishers, Dordrecht, The Netherlands, 1994, 75-87. 